\title{
Existence of solutions for damped elastic systems in Banach spaces
}

\section{Haide Gou ${ }^{*}$ and Yongxiang Li ${ }^{1}$}

\section{"Correspondence:}

842204214@qq.com

'Department of Mathematics,

Northwest Normal University,

Lanzhou, People's Republic of China

\begin{abstract}
In this article, we study the existence of mild solutions for damped elastic systems in Banach spaces. The discussion is based on the operator semigroup theory and fixed point theorem. In addition, two examples are given to illustrate our results.
\end{abstract}

MSC: $26 \mathrm{~A} 33 ; 34 \mathrm{~K} 37 ; 34 \mathrm{~A} 08 ; 34 \mathrm{~A} 37$

Keywords: Damped elastic systems; Mild solution; Equicontinuous semigroup; Existence of solutions

\section{Introduction}

In this article, we study the existence of mild solutions for the semilinear damped elastic systems in Banach spaces $E$ :

$$
\left\{\begin{array}{l}
u^{\prime \prime}(t)+\rho B u^{\prime}(t)+A u(t)=f(t, u(t)), \quad 0<t<a, \\
u(0)=u_{0} \in D(A), \quad u^{\prime}(0)=u_{1} \in E
\end{array}\right.
$$

where $A: D(A) \subset E \rightarrow E$ and $B: D(B) \subset E \rightarrow E$ are densely defined closed (possibly unbounded) linear operators on a complex Banach space $E$ and $f \in C([0, a] \times E, E)$.

In 1982, Chen and Russell [1] investigated the following linear elastic system described by the second order equation:

$$
\left\{\begin{array}{l}
u^{\prime \prime}(t)+B u^{\prime}(t)+A u(t)=0, \quad t>0 \\
u(0)=x_{0}, \quad u^{\prime}(0)=y_{0}
\end{array}\right.
$$

in a Hilbert space $H$ with inner (., ), where $A$ (the elastic operator) and $B$ (the damping operator) are positive definite self-adjoint operators in $H$. They reduced (1.2) to the first order equation in $H \times H$ :

$$
\frac{d}{d t}\left(\begin{array}{c}
A^{\frac{1}{2}} u \\
u^{\prime}
\end{array}\right)=\left(\begin{array}{cc}
0 & A^{\frac{1}{2}} \\
-A^{\frac{1}{2}} & -B
\end{array}\right)\left(\begin{array}{c}
A^{\frac{1}{2}} u \\
u^{\prime}
\end{array}\right) .
$$


Let $V=D\left(A^{\frac{1}{2}}\right), \mathcal{H}=V \times H$ with the naturally induced inner products. Then (1.2) is equivalent to the first order equation in $\mathscr{H}$

$$
\frac{d}{d t}\left(\begin{array}{c}
A^{\frac{1}{2}} u \\
u^{\prime}
\end{array}\right)=\mathcal{A}_{B}\left(\begin{array}{c}
A^{\frac{1}{2}} u \\
u^{\prime}
\end{array}\right)
$$

where

$$
\begin{aligned}
& \mathcal{A}_{B}=\left(\begin{array}{cc}
0 & I \\
-A & -B
\end{array}\right), \\
& D\left(\mathcal{A}_{B}\right)=D(A) \times\left[D\left(A^{\frac{1}{2}}\right) \cap D(B)\right] .
\end{aligned}
$$

Chen and Russell [1] conjectured that $\mathcal{A}_{B}$ is the infinitesimal generator of an analytic semigroup on $\mathscr{H}$ if

$$
D\left(A^{\frac{1}{2}}\right) \subset D(B)
$$

and either of the following two inequalities holds for some $\beta_{1}, \beta_{2}>0$ :

$$
\begin{aligned}
& \beta_{1}\left(A^{\frac{1}{2}} v, v\right) \leq(B v, v) \leq \beta_{2}\left(A^{\frac{1}{2}} v, v\right), \quad v \in D\left(A^{\frac{1}{2}}\right) \\
& \beta_{1}(A v, v) \leq\left(B^{2} v, v\right) \leq \beta_{2}(A v, v), \quad v \in D(A)
\end{aligned}
$$

The complete proofs of the two conjectures were given by Huang [2, 3]. Then, other sufficient conditions for $\mathcal{A}_{B}$ or its closure $\overline{\mathcal{A}_{B}}$ to generate an analytic or differentiable semigroup on $\mathscr{H}$ were discussed in [4-10], by choosing $B$ to be an operator comparable with $A^{\alpha}$ for $0<\alpha \leq 1$, based on an explicit matrix representation of the resolvent operator of $\mathcal{A}_{B}$ or $\overline{\mathcal{A}_{B}}$.

In [11], Fan, Li, and Chen studied the existence of mild solutions for the elastic system with structural damping in Banach spaces:

$$
\left\{\begin{array}{l}
u^{\prime \prime}(t)+\rho A u^{\prime}(t)+A^{2} u(t)=f(t, u(t)), \quad 0<t<a \\
u(0)=u_{0} \in D(A), \quad u^{\prime}(0)=u_{1} \in E
\end{array}\right.
$$

where the damping constant $\rho \geq 2$ and the nonlinearity term $f$ is Lipschitzian in the second variable. In [12], Fan and Li studied the asymptotic stability of solutions and the analyticity and exponential stability of associated semigroups for the following second order semilinear evolution equations:

$$
\left\{\begin{array}{l}
u^{\prime \prime}(t)+\rho A u^{\prime}(t)+A^{2} u(t)=0, \quad t>0, \\
u(0)=u_{0} \in D(A), \quad u^{\prime}(0)=u_{1} \in E,
\end{array}\right.
$$

where $A: D(A) \subset E \rightarrow E$ is a sectorial linear operator on a complex Banach space $E$ and $\rho>2 \cos \alpha$ for a fixed value $\alpha \in\left(0, \frac{\pi}{2}\right)$. 
In [13], Fan and Gao discussed the asymptotic behavior of solutions for the linear elastic system with structural damping:

$$
\left\{\begin{array}{l}
u^{\prime \prime}(t)+\rho A u^{\prime}(t)+A^{2} u(t)=h(t), \quad t>0 \\
u(0)=u_{0} \in D(A), \quad u^{\prime}(0)=u_{1} \in E
\end{array}\right.
$$

and the semilinear elastic system with structural damping (1.3) in Banach spaces, where $\rho>2 \cos \alpha$, for a fixed value $\alpha \in\left(0, \frac{\pi}{2}\right), A$ is a sectorial operator, $-A$ generates an analytic and exponentially stable semigroup on $E, h:[0,+\infty) \rightarrow E$ is continuous, and $f$ is Lipschitz continuous in the second variable. The discussion is based on the operator semigroup theory and some fixed point theorem.

In [14], Diagana studied the well-posedness and existence of bounded solutions to the linear elastic systems with damping:

$$
\left\{\begin{array}{l}
u^{\prime \prime}(t)+\rho B u^{\prime}(t)+A u(t)=f(t), \quad t>0, \\
u(0)=u_{0} \in D(A), \quad u^{\prime}(0)=u_{1} \in E
\end{array}\right.
$$

where $A: D(A) \subset E \rightarrow E$ and $B: D(B) \subset E \rightarrow E$ are densely defined closed (possibly unbounded) linear operators on a complex Banach space $E$ and $f: \mathbb{R}_{+} \rightarrow E$ is a continuous function.

In [15], the authors considered nonlinear evolution equations of second order in Banach spaces:

$$
\left\{\begin{array}{l}
u^{\prime \prime}(t)+\rho A u^{\prime}(t)+A^{2} u(t)=f\left(t, u(t), u_{t}\right), \quad t \in I=[0, T] \\
u(s)=\varphi(s), \quad s \leq 0, \\
u^{\prime}(0)+h(u)=\psi,
\end{array}\right.
$$

where $u$ is the unknown function defined on $I$ and, taking values in $E, u_{t}$ is the history state defined by $u_{t}:(-\infty, 0] \rightarrow E, u_{t}(s)=u(t+s), t \in I$. By means of the fixed point for condensing maps, they proved the existence and exponential decay of mild solutions.

In [16], the authors studied a class of elastic systems with structural damping:

$$
\left\{\begin{array}{l}
u^{\prime \prime}(t)+\rho A u^{\prime}(t)+A^{2} u(t)=f(t, u(t)), \quad t>0, \\
u(0)+g(u)=x_{0}, \quad u^{\prime}(0)+h(u)=y_{0},
\end{array}\right.
$$

where $A: D(A) \subset E \rightarrow E$ is a closed linear operator, $\rho \geq 2$ is a given constant, $x_{0} \in D(A)$, $y_{0} \in E$. By using the measure of noncompactness on the space of continuous functions on the half line, they established the existence of mild solutions with explicit decay rate of exponential type. Although the above discussions have been an interesting subject, the theory still remains to be developed to nonlinear case.

However, motivated by the above works, ideas and methods based on paper [14], in this paper, we investigate the existence of mild solutions for the elastic system (1.1) in complex Banach spaces. We give the expression of the solution of Problem (1.1), which is different from the expression given in article [14]. Our results presented in this paper improve and generalize many classical results $[11-14,17-20]$. 
The paper is organized as follows. In Sect. 2, we introduce some notations and recall some basic known results. In Sect. 3 we present the existence of mild solutions to Problem (1.1) in a complex Banach space. In Sect. 4, we give an example to illustrate our results.

\section{Preliminaries}

Let $E$ be a complex Banach space with the norm $\|\cdot\|$. For any constant $a>0$, denote $J=[0, a]$. Let $C(J, E)$ be the Banach space of all continuous functions from $J$ into $E$ endowed with the supremum norm $\|u\|_{C}=\sup _{t \in J}\|u(t)\|$ for every $u \in C(J, E)$. Let $\mathcal{L}(E)$ be the Banach space of all linear and bounded operators on $E$. The notations $D(L)$ and $\rho(L)$ stand respectively for the domain and resolvent of $L$, set $R(\lambda, L)=(\lambda I-L)^{-1}$ for all $\lambda \in \rho(L)$.

Definition 2.1 A $C_{0}$-semigroup $T(t)(t \geq 0)$ in $\mathrm{E}$ is said to be equicontinuous if $T(t)$ is continuous by the operator norm for every $t>0$.

Now we introduce some basic definitions and properties about Kuratowski measure of noncompactness that will be used in sequel.

Definition 2.2 ([21, 22]) The Kuratowski measure of noncompactness $\alpha(\cdot)$ defined on the bounded set $S$ of a Banach space $E$ is

$$
\alpha(S):=\inf \left\{\delta>0: S=\bigcup_{i=1}^{m} S_{i} \text { with } \operatorname{diam}\left(S_{i}\right) \leq \delta \text { for } i=1,2, \ldots, m\right\} .
$$

In this paper, we denote by $\alpha(\cdot), \alpha_{C}(\cdot)$ and the Kuratowski measure of noncompactness on the bounded set of $E, C(J, E)$, respectively. For any $D \subset C(J, E)$ and $t \in J$, set $D(t)=\{u(t) \mid u \in D\}$, then $D(t) \subset E$. If $D \subset C(J, E)$ is bounded, then $D(t)$ is bounded in $E$ and $\alpha(D(t)) \leq \alpha_{C}(D)$. For more details about the properties of the Kuratowski measure of noncompactness, we refer to the monographs [21, 22].

Definition 2.3 ([22]) Let $E$ be a Banach space, and let $S$ be a nonempty subset of $E$. A continuous mapping $Q: S \rightarrow E$ is called $k$-set-contractive if there exists a constant $k \in[0,1)$ such that, for every bounded set $\Omega \subset S$,

$$
\alpha(Q(\Omega)) \leq k \alpha(\Omega) .
$$

Lemma 2.1 ([22]) Let E be a Banach space. Assume that $\Omega \subset E$ is a bounded closed and convex set on $E$, the operator $Q: \Omega \rightarrow \Omega$ is k-set-contractive. Then $Q$ has at least one fixed point in $\Omega$.

Lemma 2.2 ([23]) Let $E$ be a Banach space, and let $D \subset E$ be bounded. Then there exists a countable set $D_{0} \subset D$ such that $\alpha(D) \leq 2 \alpha\left(D_{0}\right)$.

Lemma 2.3 ([24]) Let E be a Banach space, and let $D=\left\{u_{n}\right\} \subset C\left(\left[b_{1}, b_{2}\right], E\right)$ be a bounded and countable set for constants $-\infty<b_{1}<b_{2}<+\infty$. Then $\alpha(D(t))$ is Lebesgue integral on $\left[b_{1}, b_{2}\right]$, and

$$
\alpha\left(\left\{\int_{b_{1}}^{b_{2}} u_{n}(t) d t: n \in \mathbb{N}\right\}\right) \leq 2 \int_{b_{1}}^{b_{2}} \alpha(D(t)) d t .
$$


Lemma 2.4 ([21]) Let $E$ be a Banach space, and let $D \subset C\left(\left[b_{1}, b_{2}\right], E\right)$ be bounded and equicontinuous. Then $\alpha(D(t))$ is continuous on $\left[b_{1}, b_{2}\right]$, and

$$
\alpha_{C}(D)=\max _{t \in\left[b_{1}, b_{2}\right]} \alpha(D(t))
$$

Lemma 2.5 ([25]) Assume $f \in C(J, E)$ and that $A$ is the infinitesimal generator of $C_{0^{-}}$ semigroup $(T(t))_{t \geq 0}$. Then the inhomogeneous Cauchy problem

$$
\left\{\begin{array}{l}
u^{\prime}(t)=A u(t)+f(t), \quad t \in J, \\
u(0)=u_{0} \in D(A)
\end{array}\right.
$$

has a mild solution u given by

$$
u(t)=T(t) u_{0}+\int_{0}^{t} T(t-s) f(s) d s, \quad t \in J
$$

Thoughts and methods based on paper [13]. We consider the following linear damped elastic system:

$$
\left\{\begin{array}{l}
u^{\prime \prime}(t)+\rho B u^{\prime}(t)+A u(t)=h(t), \quad t \in J, \\
u(0)=u_{0} \in D(A), \quad u^{\prime}(0)=u_{1} \in E,
\end{array}\right.
$$

where $A: D(A) \subset E \rightarrow E$ and $B: D(B) \subset E \rightarrow E$ are densely defined closed (possibly unbounded) linear operators on a complex Banach space $E$ and $h: J \rightarrow E$.

For the second order evolution equation

$$
u^{\prime \prime}(t)+\rho B u^{\prime}(t)+A u(t)=h(t)
$$

it has the following decomposition:

$$
\left(\frac{d}{d t}+E_{1}(\rho)\right)\left(\frac{d}{d t}+E_{2}(\rho)\right) u=h(t), \quad t>0 .
$$

That is,

$$
\frac{d^{2} u}{d t^{2}}+\left(E_{1}(\rho)+E_{2}(\rho)\right) \frac{d u}{d t}+E_{1}(\rho) E_{2}(\rho) u=h(t) .
$$

It follows from (2.3) and (2.5) that

$$
E_{1}(\rho)+E_{2}(\rho)=\rho B, \quad E_{1}(\rho) E_{2}(\rho)=A .
$$

By (2.6), we have

(i) if $C(\rho)=\rho^{2} B^{2}-4 A=L^{2}(\rho)>0$, then

$$
\begin{aligned}
& E_{1}(\rho)=\frac{\rho B-\sqrt{\rho^{2} B^{2}-4 A}}{2}=\frac{\rho B-L(\rho)}{2}, \\
& E_{2}(\rho)=\frac{\rho B+\sqrt{\rho^{2} B^{2}-4 A}}{2}=\frac{\rho B+L(\rho)}{2} ;
\end{aligned}
$$


(ii) if $C(\rho)=\rho^{2} B^{2}-4 A=L^{2}(\rho)=0$, then

$$
E_{1}(\rho)=E_{2}(\rho)=\frac{\rho B}{2}
$$

(iii) if $C(\rho)=\rho^{2} B^{2}-4 A=-L^{2}(\rho)<0$, then

$$
\begin{aligned}
& E_{1}(\rho)=\frac{\rho B-\sqrt{\rho^{2} B^{2}-4 A}}{2}=\frac{\rho B-i L(\rho)}{2}, \\
& E_{2}(\rho)=\frac{\rho B+\sqrt{\rho^{2} B^{2}-4 A}}{2}=\frac{\rho B+i L(\rho)}{2} .
\end{aligned}
$$

Remark 2.1 In order to study the existence to Eq. (1.1), we will make use of the above linear operator which links both $A$ and $B: C(\rho)=\rho^{2} B^{2}-4 A=L^{2}(\rho)$ with $D(C(\rho))=D\left(B^{2}\right) \cap D(A)$. In the following discussion, we will focus on the following cases: $C(\rho)=L^{2}(\rho)>0$ and $C(\rho)=L^{2}(\rho)=0$ for densely closed linear operator $L(\rho): D(L(\rho)) \subset E \rightarrow E$. Obviously, $C(\rho)=0$ corresponds to the case studied in papers $[11,12]$. For more details, see [14].

Lemma 2.6 Assume that there exists a densely defined closed linear operator $L(\rho)$ : $D(L(\rho)) \subset E \rightarrow E$ such that $u_{0} \in D(L(\rho)) \cap D(B)$ and $C(\rho)=\rho^{2} B^{2}-4 A=L^{2}(\rho)$ and $B L(\rho)=L(\rho) B$. Let $h \in C(J, E),-E_{1}(\rho)$ and $-E_{2}(\rho)$ be respectively the infinitesimal generators of $C_{0}$-semigroups $T_{1}(t)(t \geq 0)$ and $T_{2}(t)(t \geq 0)$. Then Problem (2.2) has a unique solution given by

$$
\begin{aligned}
u(t)= & T_{2}(t) u_{0}+\int_{0}^{t} T_{2}(t-s) T_{1}(s)\left(u_{1}+E_{2}(\rho) u_{0}\right) d s \\
& +\int_{0}^{t} \int_{0}^{s} T_{2}(t-s) T_{1}(s-\tau) h(\tau) d \tau d s
\end{aligned}
$$

where $E_{1}(\rho), E_{2}(\rho)$ were defined in (2.7).

Proof Let

$$
\frac{d u}{d t}+E_{2}(\rho) u=v(t), \quad t \in J
$$

which means

$$
v_{0}:=v(0)=u_{1}+E_{2}(\rho) u_{0} .
$$

So we reduce the linear elastic system (2.2) to the following two abstract Cauchy problems in a Banach space $E$ :

$$
\left\{\begin{array}{l}
\frac{d v}{d t}+E_{1}(\rho) v=h(t), \quad t \in J \\
v(0)=v_{0}
\end{array}\right.
$$

and

$$
\left\{\begin{array}{l}
\frac{d u}{d t}+E_{2}(\rho) u=v(t), \quad t \in J \\
u(0)=u_{0} .
\end{array}\right.
$$


It is clear that (2.9) and (2.10) are linear inhomogeneous initial value problems for $-E_{1}(\rho)$ and $-E_{2}(\rho)$, respectively. Thus, by operator semigroups theory [11], $-E_{1}(\rho)$ and $-E_{2}(\rho)$ are infinitesimal generators of $C_{0}$-semigroups, which implies that initial value problems (2.9) and (2.10) are well posed.

Thus using Lemma 2.6, if $h \in C(J, E)$, Problem (2.9) has a mild solution $v$ given by

$$
v(t)=T_{1}(t) v_{0}+\int_{0}^{t} T_{1}(t-s) h(s) d s .
$$

Similarly, if $v \in C(J, E)$, then the mild solution of Problem (2.10) is expressed by

$$
u(t)=T_{2}(t) u_{0}+\int_{0}^{t} T_{2}(t-s) v(s) d s
$$

Substituting (2.11) into (2.12), we get

$$
\begin{aligned}
u(t)= & T_{2}(t) u_{0}+\int_{0}^{t} T_{2}(t-s) T_{1}(s)\left(u_{1}+E_{2}(\rho) u_{0}\right) d s \\
& +\int_{0}^{t} \int_{0}^{s} T_{2}(t-s) T_{1}(s-\tau) h(\tau) d \tau d s .
\end{aligned}
$$

Throughout this paper, we assume that $-E_{1}(\rho)$ and $-E_{2}(\rho)$ generate $C_{0}$-semigroups $T_{1}(t)$ $(t \geq 0)$ and $T_{2}(t)(t \geq 0)$ on $E$, respectively.

Based on the above discussion, motivated by the definition of mild solutions in [13], we give the definition of a mild solution of Problem (1.1) as follows.

Definition 2.4 Let $f \in C(J \times E, E),-E_{1}(\rho)$ and $-E_{2}(\rho)$ be respectively the infinitesimal generators of $C_{0}$-semigroups $T_{1}(t)(t \geq 0)$ and $T_{2}(t)(t \geq 0)$. A function $u: J \rightarrow E$ is said to be a mild solution of Problem (1.1) if $u(0)=u_{0}$ and

$$
\begin{aligned}
u(t)= & T_{2}(t) u_{0}+\int_{0}^{t} T_{2}(t-s) T_{1}(s)\left(u_{1}+E_{2}(\rho) u_{0}\right) d s \\
& +\int_{0}^{t} \int_{0}^{s} T_{2}(t-s) T_{1}(s-\tau) f(\tau, u(\tau)) d \tau d s
\end{aligned}
$$

where $E_{1}(\rho), E_{2}(\rho)$ were defined in (2.7).

\section{Main results}

To obtain the existence of a mild solution for Problem (1.1), we introduce the following hypotheses:

(H1) Assume that there exists a densely defined closed linear operator $L(\rho): D(L(\rho)) \subset E \rightarrow E$ such that $u_{0} \in D(L(\rho)) \cap D(B)$ and

$$
C(\rho)=\rho^{2} B^{2}-4 A=L^{2}(\rho), \quad B L(\rho)=L(\rho) B .
$$

(H2) The nonlinear function $f: J \times E \rightarrow E$ is continuous, there exist a Lebesgue integrable function $\varphi \in L^{\infty}\left(J, \mathbb{R}^{+}\right)$and a nondecreasing continuous function 
$\Psi:[0,+\infty) \rightarrow(0,+\infty)$ such that

$$
\|f(t, u)\| \leq \varphi(t) \Psi(\|u\|)
$$

for all $u \in C(J, E)$.

(H3) There exists a positive constant $L$ such that $L<\frac{1}{4 a^{2} M_{1} M_{2}}$ for any countable set $D \subset E$,

$$
\alpha(f(t, D)) \leq L \alpha(D), \quad t \in J
$$

(H4) The $C_{0}$-semigroups $T_{2}(t)(t \geq 0)$ and $T_{1}(t)(t \geq 0)$ are equicontinuous for $t>0$.

Theorem 3.1 Assume that $-E_{1}(\rho)$ and $-E_{2}(\rho)$ are respectively the infinitesimal generators of $C_{0}$-semigroups $T_{1}(t)(t \geq 0)$ and $T_{2}(t)(t \geq 0)$. If conditions $(\mathrm{H} 1)-(\mathrm{H} 4)$ hold and

$$
a^{2} M_{1} M_{2}\|\varphi\|_{L^{\infty}\left(J, \mathbb{R}^{+}\right)} \liminf _{n \rightarrow+\infty} \frac{\Psi(n)}{n}<1,
$$

then, for every $u_{0} \in D(L(\rho)) \cap D(B), u_{1} \in E$, Problem (1.1) has at least one mild solution $u \in C(J, E)$.

Proof Define the operator $\mathcal{F}: C(J, E) \rightarrow C(J, E)$ by

$$
\begin{aligned}
\mathcal{F} u(t)= & T_{2}(t) u_{0}+\int_{0}^{t} T_{2}(t-s) T_{1}(s)\left(u_{1}+E_{2}(\rho) u_{0}\right) d s \\
& +\int_{0}^{t} \int_{0}^{s} T_{2}(t-s) T_{1}(s-\tau) f(\tau, u(\tau)) d \tau d s .
\end{aligned}
$$

It is easy to see that the operator $\mathcal{F}$ is well defined on $C(J, E)$. From Definition 2.4, one can easily see that the mild solution of Problem (1.1) is equivalent to a fixed point of the operator $\mathcal{F}$ defined by (3.2). Next, we will prove that the operator $\mathcal{F}$ has at least one fixed point.

Let $\Omega_{R}=\left\{u \in C(J, E):\|u\|_{C} \leq R\right\}$, then $\Omega_{R}$ is a bounded closed and convex set in $C(J, E)$. Note that $T_{1}(t)(t \geq 0)$ and $T_{2}(t)(t \geq 0)$ are $C_{0}$-semigroups on $E$, then there exist $M_{1} \geq 1$ and $M_{2} \geq 1$ such that

$$
M_{1}=\sup _{t \in J}\left\|T_{1}(t)\right\|_{\mathcal{L}(E)}, \quad M_{2}=\sup _{t \in J}\left\|T_{2}(t)\right\|_{\mathcal{L}(E)}
$$

Firstly, we prove that there exists a constant $R>0$ such that $\mathcal{F}\left(\Omega_{R}\right) \subset \Omega_{R}$. Assume to the contrary that, for each $n \in \mathbb{N}$, there exists a sequence $\left\{u_{n}\right\}_{n=1}^{\infty} \subset \Omega_{n}$ with $\left\|u_{n}\right\|_{C} \leq n$ but $\left\|\mathcal{F}\left(u_{n}\right)\right\|_{C}>n$. Then, by (3.2) and assumptions (H1), (H2), we have

$$
\begin{aligned}
\left\|\left(\mathcal{F} u_{n}\right)(t)\right\| & \leq M_{2}\left\|u_{0}\right\|+M_{1} M_{2} a\left\|u_{1}+E_{2}(\rho) u_{0}\right\|+M_{1} M_{2} \int_{0}^{t} \int_{0}^{s}\left\|f\left(\tau, u_{n}(\tau)\right)\right\| d \tau d s \\
& \leq M_{2}\left\|u_{0}\right\|+M_{1} M_{2} a\left\|u_{1}+E_{2}(\rho) u_{0}\right\|+M_{1} M_{2} a^{2} \Psi\left(\left\|u_{n}\right\|\right)\|\varphi\|_{L^{\infty}\left(, \mathbb{R}^{+}\right)} .
\end{aligned}
$$


Hence,

$$
\begin{aligned}
1 & <\frac{1}{n}\left\|\left(\mathcal{F} u_{n}\right)(t)\right\| \\
& \leq \frac{1}{n}\left(M_{2}\left\|u_{0}\right\|+M_{1} M_{2} a\left\|u_{1}+E_{2}(\rho) u_{0}\right\|\right)+M_{1} M_{2} a^{2}\|\varphi\|_{L^{\infty}\left(J, \mathbb{R}^{+}\right)} \frac{\Psi(n)}{n} .
\end{aligned}
$$

Passing to the limit in (3.4), we get a contradiction. Hence, for some positive number $R, \mathcal{F}\left(\Omega_{R}\right) \subset \Omega_{R}$.

Next, we prove that $\mathcal{F}$ is continuous in $\Omega_{R}$. To this end, let $u_{n} \in \Omega_{R}$ be a sequence such that $u_{n} \rightarrow u$ in $\Omega_{R}$. By the continuity of nonlinear $\operatorname{term} f$ with respect to the second variable, for each $s \in J$, we have

$$
f\left(s, u_{n}(s)\right) \rightarrow f(s, u(s)), \quad n \rightarrow \infty
$$

that is, for all $\epsilon>0$, there exists $N$, when $n>N$, we have

$$
\left\|f\left(s, u_{n}(s)\right)-f(s, u(s))\right\| \leq \epsilon .
$$

Now, we have

$$
\begin{aligned}
\left\|\left(\mathcal{F} u_{n}\right)(t)-(\mathcal{F} u)(t)\right\| & \leq M_{1} M_{2} \int_{0}^{t} \int_{0}^{s}\left\|f\left(\tau, u_{n}(\tau)\right)-f(\tau, u(\tau))\right\| d \tau d s \\
& \leq M_{1} M_{2} a^{2}\left\|f\left(\tau, u_{n}(\tau)\right)-f(\tau, u(\tau))\right\| .
\end{aligned}
$$

So, when $n>N$, we have

$$
\left\|\mathcal{F} u_{n}-\mathcal{F} u\right\|_{C} \leq M_{1} M_{2} a^{2} \epsilon,
$$

which means that $\mathcal{F}$ defined by (3.2) is continuous in $\Omega_{R}$.

In the following, we demonstrate that the operator $\mathcal{F}: \Omega_{R} \rightarrow \Omega_{R}$ is equicontinuous. For any $u \in \Omega_{R}$ and $0<t^{\prime}<t^{\prime \prime} \leq a$, we obtain that

$$
\begin{aligned}
\left\|\left(\mathcal{F}_{2} u\right)\left(t^{\prime \prime}\right)-\left(\mathcal{F}_{2} u\right)\left(t^{\prime}\right)\right\| \\
\leq\left\|T_{2}\left(t^{\prime \prime}\right) u_{0}-T_{2}\left(t^{\prime}\right) u_{0}\right\| \\
+\left\|\int_{0}^{t^{\prime}}\left[T_{2}\left(t^{\prime \prime}-s\right)-T_{2}\left(t^{\prime}-s\right)\right] T_{1}(s)\left(u_{1}+E_{2}(\rho) u_{0}\right) d s\right\| \\
+\left\|\int_{t^{\prime}}^{t^{\prime \prime}} T_{2}\left(t^{\prime \prime}-s\right) T_{1}(s)\left(u_{1}+E_{2}(\rho) u_{0}\right) d s\right\| \\
+\left\|\int_{0}^{t^{\prime}} \int_{0}^{s}\left[T_{2}\left(t^{\prime \prime}-s\right)-T_{2}\left(t^{\prime}-s\right)\right] \times T_{1}(s-\tau) f(\tau, u(\tau)) d \tau d s\right\| \\
\quad\left\|\int_{t^{\prime}}^{t^{\prime \prime}} \int_{0}^{s} T_{2}\left(t^{\prime \prime}-s\right) \times T_{1}(s-\tau) f(\tau, u(\tau)) d \tau d s\right\| \\
:=I_{1}+I_{2}+I_{3}+I_{4}+I_{5},
\end{aligned}
$$


where

$$
\begin{aligned}
& I_{1}=\left\|T_{2}\left(t^{\prime \prime}\right) u_{0}-T_{2}\left(t^{\prime}\right) u_{0}\right\|, \\
& I_{2}=\left\|\int_{0}^{t^{\prime}}\left[T_{2}\left(t^{\prime \prime}-s\right)-T_{2}\left(t^{\prime}-s\right)\right] T_{1}(s)\left(u_{1}+E_{2}(\rho) u_{0}\right) d s\right\|, \\
& I_{3}=\left\|\int_{t^{\prime}}^{t^{\prime \prime}} T_{2}\left(t^{\prime \prime}-s\right) T_{1}(s)\left(u_{1}+E_{2}(\rho) u_{0}\right) d s\right\|, \\
& I_{4}=\left\|\int_{0}^{t^{\prime}} \int_{0}^{s}\left[T_{2}\left(t^{\prime \prime}-s\right)-T_{2}\left(t^{\prime}-s\right)\right] \times T_{1}(s-\tau) f(\tau, u(\tau)) d \tau d s\right\| \\
& I_{5}=\left\|\int_{t^{\prime}}^{t^{\prime \prime}} \int_{0}^{s} T_{2}\left(t^{\prime \prime}-s\right) \times T_{1}(s-\tau) f(\tau, u(\tau)) d \tau d s\right\| .
\end{aligned}
$$

In fact, we only need to check whether $I_{1}, I_{2}, I_{3}, I_{4}$, and $I_{5}$ tend to 0 independently of $u \in \Omega_{R}$ when $t^{\prime \prime}-t^{\prime} \rightarrow 0$.

Note that the function $T_{2}(t) u_{0}$ is continuous for $t \geq 0$. Thus, $T_{2}(t) u_{0}$ is uniformly continuous on $J$ and thus $\lim _{t^{\prime \prime} \rightarrow t^{\prime}} I_{1}=0$.

For $I_{2}$, we have

$$
\begin{aligned}
I_{2} & \leq \int_{0}^{t^{\prime}}\left\|T_{2}\left(t^{\prime \prime}-s\right)-T_{2}\left(t^{\prime}-s\right)\right\|_{\mathcal{L}(E)} \times\left\|T_{1}(s)\right\|_{\mathcal{L}(E)}\left\|u_{1}+E_{2}(\rho) u_{0}\right\| d s \\
& \leq M_{1}\left\|u_{1}+E_{2}(\rho) u_{0}\right\| \int_{0}^{t^{\prime}}\left\|T_{2}\left(t^{\prime \prime}-s\right)-T_{2}\left(t^{\prime}-s\right)\right\|_{\mathcal{L}(E)} d s .
\end{aligned}
$$

Therefore the continuity of the functions $t \mapsto\left\|T_{1}(t)\right\|$ and $t \mapsto\left\|T_{2}(t)\right\|$ for $t \in J$ allows us to conclude that $\lim _{t^{\prime \prime} \rightarrow t^{\prime}} I_{2}=0$.

For $I_{4}$, we have

$$
\begin{aligned}
I_{4} & \leq \int_{0}^{t^{\prime}} \int_{0}^{s}\left\|T_{2}\left(t^{\prime \prime}-s\right)-T_{2}\left(t^{\prime}-s\right)\right\|_{\mathcal{L}(E)} \times\left\|T_{1}(s-\tau)\right\|_{\mathcal{L}(E)}\|f(\tau, u(\tau))\| d \tau d s \\
& \leq M_{1} a \Psi(R)\|u\|_{L^{\infty}(J, E)} \times \int_{0}^{t^{\prime}}\left\|T_{2}\left(t^{\prime \prime}-s\right)-T_{2}\left(t^{\prime}-s\right)\right\|_{\mathcal{L}(E)} d s .
\end{aligned}
$$

Consequently, $\lim _{t^{\prime \prime} \rightarrow t^{\prime}} I_{4}=0$.

For $I_{3}, I_{5}$, we ha

$$
\begin{aligned}
& I_{3} \leq M_{1} M_{2}\left\|u_{1}+E_{2}(\rho) u_{0}\right\| \cdot\left|t^{\prime \prime}-t^{\prime}\right|, \\
& I_{5} \leq M_{1} M_{2} \Psi(R)\|u\|_{L^{\infty}(J, E)}\left|t^{\prime \prime}-t^{\prime}\right| .
\end{aligned}
$$

Hence, $\lim _{t^{\prime \prime} \rightarrow t^{\prime}} I_{3}=\lim _{t^{\prime \prime} \rightarrow t^{\prime}} I_{5}=0$.

As a result, $\left\|(\mathcal{F} u)\left(t^{\prime \prime}\right)-(\mathcal{F} u)\left(t^{\prime}\right)\right\|$ tends to 0 independently of $u \in \Omega_{R}$ as $t^{\prime \prime}-t^{\prime} \rightarrow 0$, which means that $\mathcal{F}: \Omega_{R} \rightarrow \Omega_{R}$ is equicontinuous.

Now, we show that the operator $\mathcal{F}$ is $k$-set-contractive. For any bounded $D \subset \Omega_{R}$, by Lemma 2.2, we know that there exists a countable set $D_{0}=\left\{u_{n}\right\} \subset D$ such that

$$
\alpha(\mathcal{F}(D))_{C} \leq 2 \alpha\left(\mathcal{F}\left(D_{0}\right)\right)_{C}
$$


Since $\mathcal{F}\left(D_{0}\right) \subset \mathcal{F}\left(\Omega_{R}\right)$ is bounded and equicontinuous, we know from Lemma 2.4 that

$$
\alpha\left(\mathcal{F}\left(D_{0}\right)\right)_{C}=\max _{t \in J} \alpha\left(\mathcal{F}\left(D_{0}\right)(t)\right)
$$

For every $t \in J$, by Lemma 2.3, assumption (H3), and (3.2), we have

$$
\begin{aligned}
\alpha\left(\mathcal{F}\left(D_{0}\right)(t)\right)= & \alpha\left(\left\{T_{2}(t) u_{0}+\int_{0}^{t} T_{2}(t-s) T_{1}(s)\left(u_{1}+E_{2}(\rho) u_{0}\right) d s\right.\right. \\
& \left.\left.+\int_{0}^{t} \int_{0}^{s} T_{2}(t-s) T_{1}(s-\tau) f\left(\tau, u_{n}(\tau)\right) d \tau d s\right\}\right) \\
\leq & 2 M_{1} M_{2} a \int_{0}^{t} \alpha\left(\left\{f\left(\tau, u_{n}(\tau)\right)\right\}\right) d \tau \\
\leq & 2 M_{1} M_{2} a \int_{0}^{t} L \alpha\left(D_{0}(s)\right) d s \\
\leq & 2 M_{1} M_{2} L a^{2} \alpha(D)_{C} .
\end{aligned}
$$

Therefore, from (3.7) and (3.9) we know that

$$
\alpha(\mathcal{F}(D))_{C} \leq 4 M_{1} M_{2} L a^{2} \alpha(D)_{C}
$$

And from Definition 2.3 we know that the operator $\mathcal{F}: \Omega_{R} \rightarrow \Omega_{R}$ is $k$-set-contractive. It follows from Lemma 2.1 that $\mathcal{F}$ has at least one fixed point $u \in \Omega_{R}$, which is just a mild solution of Problem (1.1). This completes the proof.

Remark 3.1 The analytic semigroup and differentiable semigroup are equicontinuous semigroups [25]. In the application of partial differential equations, such as parabolic and strongly damped wave equations, the corresponding solution semigroup is analytic semigroup. Therefore, Theorem 3.1 has a broad applicability.

Remark 3.2 In the case $C(\rho)=-L^{2}(\rho)$, the expression of a mild solution for Problem (1.1) and the conclusion of Theorem 3.1 are correct and meaningful in complex Banach spaces.

\section{Examples}

Let $\Omega \subset \mathbb{R}^{N}$ be an open bounded set with sufficiently smooth boundary $\partial \Omega$, and let $E=$ $L^{p}(\Omega)$. Then $E$ is a Banach space equipped with the $L^{p}$-norm $\|\cdot\|_{p}$.

Example 4.1 Let $p=2$. We consider the following damping elastic system:

$$
\left\{\begin{array}{l}
\frac{\partial^{2} u(t, x)}{\partial t^{2}}-2 \gamma \Delta \frac{\partial u(t, x)}{\partial t}+\Delta^{2} u(t, x)=\frac{1}{5} \sin u(t, x), \quad(t, x) \in J \times \Omega, \\
\Delta u(t, x)=u(t, x)=0, \quad(t, x) \in J \times \partial \Omega, \\
u(0, x)=u_{0}(x), \quad \frac{\partial}{\partial t} u(0, x)=u_{1}(x), \quad x \in \Omega,
\end{array}\right.
$$

where $\gamma=\rho \geq 1$ is constant, $\Delta$ stands for the Laplace operator in the space variable $x$, $J=[0,1]$. We define the linear operators $A$ and $B$ in $E$ by

$$
A u=\Delta^{2} u, \quad u \in D(A)=D\left(\Delta^{2}\right)=\left\{u \in H^{4}(\Omega): \Delta u=u=0 \text { on } \partial \Omega\right\},
$$




$$
B u=-2 \Delta u, \quad u \in D(B)=H_{0}^{1}(\Omega) \cap H_{0}^{2}(\Omega) .
$$

Clearly, $C(\rho)=\rho^{2} B^{2}-4 A=4 \Delta^{2}\left(\rho^{2}-1\right)=L^{2}$, where $L=2 \Delta\left(\rho^{2}-1\right)^{\frac{1}{2}}$. It is clear that $B L=$ $L B$. Further,

$$
E_{1}(\rho)=-\left(\rho+\left(\rho^{2}-1\right)^{\frac{1}{2}}\right) \Delta=-\sigma_{1} \Delta, \quad E_{2}(\rho)=-\left(\rho-\left(\rho^{2}-1\right)^{\frac{1}{2}}\right) \Delta=-\sigma_{2} \Delta
$$

where $\sigma_{1}=\left(\rho+\left(\rho^{2}-1\right)^{\frac{1}{2}}\right), \sigma_{2}=\left(\rho-\left(\rho^{2}-1\right)^{\frac{1}{2}}\right), E_{1}(\rho)$ and $E_{2}(\rho)$ are invertible bounded linear operators on $L^{2}(\Omega)$ for all $\rho>0$.

Since $\Delta$ is the infinitesimal generator of $C_{0}$-semigroup $T(t)_{t \geq 0}$, furthermore, for any $\rho \geq$ 1 , (4.2) yields $\sigma_{1}>0, \sigma_{2}>0$. Thus, by operator semigroup theory [25], $-E_{1}(\rho)=\sigma_{1} \Delta$ and $-E_{2}(\rho)=\sigma_{2} \Delta$ are the infinitesimal generators of equicontinuous $C_{0}$-semigroups $T_{1}(t)_{t \geq 0}$ and $T_{2}(t)_{t \geq 0}$ on $L^{2}(\Omega)$, respectively. It follows that

$$
T_{1}(t)=T\left(\sigma_{1} t\right), \quad T_{2}(t)=T\left(\sigma_{2} t\right), \quad t \geq 0,
$$

which is exponentially stable, i.e.,

$$
\left\|T_{1}(t)\right\| \leq e^{-\lambda_{1} \sigma_{1} t}, \quad\left\|T_{2}(t)\right\| \leq e^{-\lambda_{1} \sigma_{2} t}
$$

with $\lambda_{1}$ being the first eigenvalue of $\Delta$.

Let $u(t)=u(t, \cdot), f(t, u(t))=\frac{1}{5} \sin u(t, x)$, then Problem (4.1) can be reformulated as the following abstract second order evolution equation in $E$ :

$$
\left\{\begin{array}{l}
u^{\prime \prime}(t)+\rho B u^{\prime}(t)+A u(t)=f(t, u(t)), \quad t \in J \\
u(0)=u_{0}, \quad u^{\prime}(0)=u_{1} .
\end{array}\right.
$$

In order to solve Problem (4.1), we also need the following assumptions:

(1) $u_{0} \in D(L) \cap D(B), u_{1} \in L^{2}(\Omega)$.

(2) The partial derivative $f_{u}^{\prime}(t, x, u)$ is continuous.

Theorem 4.1 If assumptions (1) and (2) are satisfied, then Problem (4.1) has a mild solution $u \in C\left(J, L^{2}(\Omega)\right)$.

Proof Since $f(t, x, u(t, x))=\frac{1}{5} \sin u(t, x)$ is continuous on $[0,1] \times[0,+\infty) \times E$ and satisfying

$$
\begin{aligned}
& \left|f_{u}^{\prime}(t, x, u)\right|=\frac{1}{5}|\cos u(t, x)| \leq \frac{1}{5}, \quad(t, x, u) \in[0,1] \times[0,+\infty) \times E \\
& f(t, x, 0)=\sin 0=0, \quad(t, x) \in[0,1] \times[0,+\infty) .
\end{aligned}
$$

From (4.4), for $u \in E$, we have

$$
\begin{aligned}
& \|f(t, x, u)\| \leq \frac{1}{5}\|u\|, \quad(t, x) \in[0,1] \times[0,+\infty), \\
& \alpha(f(t, D)) \leq \frac{1}{5} \alpha(D), \quad t \in J .
\end{aligned}
$$


Now take $M_{1}=M_{2}=1$, we calculate

$$
\begin{aligned}
& a^{2} M_{1} M_{2}\|\varphi\|_{L^{\infty}\left(J, \mathbb{R}^{+}\right)} \liminf _{n \rightarrow+\infty} \frac{\Psi(n)}{n}=\frac{1}{5}<1, \\
& L<\frac{1}{4 a^{2} M_{1} M_{2}}=\frac{1}{4} .
\end{aligned}
$$

From all the assumptions, it is easily seen that the conditions in Theorem 3.1 are satisfied. Hence, by Theorem 3.1, Problem (4.3) has a mild solution $u \in C(J, E)$, which means $u$ is a mild solution for Problem (4.1).

Example 4.2 Let $p \in[2, \infty)$. Consider the following damping elastic system:

$$
\left\{\begin{array}{l}
\frac{\partial^{2} u(t, x)}{\partial t^{2}}+2 \rho \Delta \frac{\partial u(t, x)}{\partial t}+\Delta u(t, x)=\frac{1}{5} \sin u(t, x), \quad(t, x) \in J \times \Omega, \\
u(t, x)=0, \quad(t, x) \in J \times \partial \Omega, \\
u(0, x)=u_{0}(x), \quad \frac{\partial}{\partial t} u(0, x)=u_{1}(x), \quad x \in \Omega,
\end{array}\right.
$$

where $\rho>0$ is constant, the function $f: J \times \Omega \times E \rightarrow E$ is continuous, and $\Delta$ stands for the Laplace operator in the space variable $x, J=[0,1]$. We define the linear operators $A$ and $B$ in $E$ by

$$
\begin{aligned}
& A u=\Delta u, \quad u \in D(A)=W^{2, p}(\Omega) \cap W_{0}^{1, p}(\Omega), \\
& B u=2 \Delta u, \quad u \in D(B)=W^{2, p}(\Omega) \cap W_{0}^{1, p}(\Omega) .
\end{aligned}
$$

Clearly, $C(\rho)=\rho^{2} B^{2}-4 A=4\left(\rho^{2} \Delta^{2}-\Delta\right)=L^{2}$, where $L=2\left(\rho^{2} \Delta^{2}-\Delta\right)^{\frac{1}{2}}$. It is clear that $B L=L B$. Further,

$$
E_{1}(\rho)=R_{1}(\rho) \Delta, \quad E_{2}(\rho)=R_{2}(\rho) \Delta,
$$

where $R_{1}(\rho)=\left[\rho I-\left(\rho^{2} I+(-\Delta)^{-1}\right)^{\frac{1}{2}}\right]$ and $R_{2}(\rho)=\left[\rho I+\left(\rho^{2} I+(-\Delta)^{-1}\right)^{\frac{1}{2}}\right]$ are invertible bounded linear operators on $L^{p}(\Omega)$ for all $\rho>0$.

Moreover,

$$
-R_{1}^{-1}(\rho)\left(-E_{1}(\rho)\right)=-R_{2}^{-1}(\rho)\left(-E_{2}(\rho)\right)=\Delta
$$

is the infinitesimal generator of a $C_{0}$-semigroup as $T(t)(t \geq 0)$ on $L^{p}(\Omega)$ and $-R_{1}^{-1}(\rho)$, $-R_{2}^{-1}(\rho)$ are invertible. Thus, by operator semigroup theory [25], $-E_{1}(\rho)=-R_{1}(\rho) \Delta$ and $-E_{2}(\rho)=-R_{2}(\rho) \Delta$ generate positive $C_{0}$-semigroups $T_{1}(t)(t \geq 0)$ and $T_{2}(t)(t \geq 0)$ on $L^{p}(\Omega)$.

Let $u(t)=u(t, \cdot), f(t, u(t))=\frac{1}{5} \sin u(t, x)$, then Problem (4.5) can be reformulated as Eqs. (4.3). In order to solve Problem (4.5), we also need the following assumptions:

(3) $u_{0} \in D(L) \cap D(B), u_{1} \in L^{p}(\Omega)$

(4) The partial derivative $f_{t}^{\prime}(t, x, u)$ is continuous.

Theorem 4.2 If assumptions (3) and (4) are satisfied, then Problem (4.5) has a mild solution $u \in C\left(J, L^{p}(\Omega)\right)$.

Proof The proof is similar to that of Theorem 4.1. Here, we omit it. 


\section{Conclusions}

This paper investigates the existence of mild solutions for damped elastic systems in Banach spaces by the fixed point theorem and operator semigroup theory. Our results presented in this paper improve and generalize many classical results [11-14]. For future work we will focus on investigating the asymptotic stability of solutions and the analyticity and exponential stability of the associated semigroup for a damping elastic system in Banach spaces.

\section{Acknowledgements}

The authors would like to thank the referees for their useful suggestions which have significantly improved the paper.

\section{Funding}

The authors are supported financially by the National Natural Science Foundation of China (11661071).

\section{Availability of data and materials}

Data sharing not applicable to this article as no datasets were generated or analysed during the current study.

\section{Ethics approval and consent to participate}

Not applicable.

\section{Competing interests}

The authors declare that there is no conflict of interests regarding the publication of this paper.

\section{Authors' contributions}

All authors contributed equally and significantly in writing this article. All authors read and approved the final manuscript.

\section{Publisher's Note}

Springer Nature remains neutral with regard to jurisdictional claims in published maps and institutional affiliations.

Received: 30 October 2018 Accepted: 7 May 2019 Published online: 16 May 2019

\section{References}

1. Chen, G., Russell, D.L.: A mathematical model for linear elastic systems with structural damping. Q. Appl. Math. 39(4), 433-454 (1982)

2. Huang, F.L.: On the holomorphic property of the semigroup associated with linear elastic systems with structural damping. Acta Math. Sci. 5(3), 271-277 (1985)

3. Huang, F.: A problem for linear elastic systems with structural damping. Acta Math. Sci. 6(1), 101-107 (1986) (Chinese)

4. Chen, S., Triggiani, R.: Proof of extensions of two conjectures on structural damping for elastic systems: the systems: the case $\frac{1}{2} \leq \alpha \leq 1$. Pac. J. Math. 136(1), 15-55 (1989)

5. Chen, S., Triggiani, R.: Gevrey class semigroups arising from elastic systems with gentle dissipation: the case $0<\alpha<\frac{1}{2}$. Proc. Am. Math. Soc. 110(2), 401-415 (1990)

6. Huang, F.L:: On the mathematical model for linear elastic systems with analytic damping. SIAM J. Control Optim. 26(3), 714-724 (1988)

7. Liu, K., Liu, Z.: Analyticity and differentiability of semigroups associated with elastic systems with damping and gyroscopic forces. J. Differ. Equ. 141(2), 340-355 (1997)

8. Huang, F.L., Liu, K.S.: Holomorphic property and exponential stability of the semigroup associated with linear elastic systems with damping. Ann. Differ. Equ. 4(4), 411-424 (1988)

9. Huang, F.L., Huang, Y.Z., Guo, F.M.: Holomorphic and differentiable properties of the $C_{0}$-semigroup associated with the Euler-Bernoulli beam equations with structural damping. Sci. China Ser. A 35(5), 547-560 (1992)

10. Huang, F.L., Liu, K.S., Chen, G.: Differentiability of the semigroup associated with a structural damping model. In: Proceedings of the 28th IEEE Conference on Decision and Control (IEEE-CDC 1989), Tampa, Fla, USA, pp. 2034-2038 (1989)

11. Fan, H., Li, Y., Chen, P.: Existence of mild solutions for the elastic systems with structural damping in Banach spaces. Abstr. Appl. Anal. 2013, Article ID 746893 (2013)

12. Fan, H., Li, Y.: Analyticity and exponential stability of semigroup for elastic systems with structural damping in Banach spaces. J. Math. Anal. Appl. 410, 316-322 (2014)

13. Fan, H., Gao, F.: Asymptotic stability of solutions to elastic systems with structural damping. Electron. J. Differ. Equ. $2014,245,9(2014)$

14. Diagana, T.: Well-posedness for some damped elastic systems in Banach spaces. Appl. Math. Lett. 71, 74-80 (2017)

15. Luong, V.T., Tung, N.T.: Exponential decay for elastic systems with structural damping and infinite delay. Appl. Anal. (2018). https://doi.org/10.1080/00036811.2018.1484907

16. Luong, V.T., Tung, N.T.: Decay mild solutions for elastic systems with structural damping involving nonlocal conditions. Vestn. St. Petersbg. Univ., Math. 50(1), 55-67 (2017)

17. Chen, P.Y., Zhang, X.P., Li, Y.X.: Approximation technique for fractional evolution equations with nonlocal integral conditions. Mediterr. J. Math. 14(6), 214-226 (2017) 
18. Chen, P.Y., Zhang, X.P., Li, Y.X.: Study on fractional non-autonomous evolution equations with delay. Comput. Math. Appl. 73(5), 794-803 (2017)

19. Chen, P.Y., Li, Y.X.: A blow up alternative result for fractional nonautonomous evolution equation of Volterra type. Commun. Pure Appl. Anal. 17(5), 1975-1992 (2018)

20. Chen, P.Y., Zhang, X.P., Li, Y.X.: Fractional non-autonomous evolution equation with nonlocal conditions. J. Pseudo-Differ. Oper. Appl. (2018). https://doi.org/10.1007/s11868-018-0257-9

21. Banas̀, J., Goebel, K.: Measures of Noncompactness in Banach Spaces. Lecture Notes in Pure and Applied Mathematics, vol. 60. Dekker, New York (1980)

22. Deimling, K.: Nonlinear Functional Analysis. Springer, New York (1985)

23. Li, Y.: Existence of solutions of initial value problems for abstract semilinear evolution equations. Acta Math. Sin. 48, 1089-1094 (2005) (in Chinese)

24. Heinz, H.P.: On the behaviour of measure of noncompactness with respect to differentiation and integration of vector-valued functions. Nonlinear Anal. 7, 1351-1371 (1983)

25. Pazy, A.: Semigroups of Linear Operators and Applications to Partial Differential Equations. Springer, Berlin (1983)

Submit your manuscript to a SpringerOpen ${ }^{0}$ journal and benefit from:

- Convenient online submission

- Rigorous peer review

- Open access: articles freely available online

- High visibility within the field

- Retaining the copyright to your article

Submit your next manuscript at $\boldsymbol{\nabla}$ springeropen.com 